\title{
Acute limb ischemia in a patient with Covid-19 pneumonia: a case report
}

\author{
Ulfah Kartikasari $^{1,2}$ (D) Susanthy Djajalaksana ${ }^{1,2} \cdot$ Heny Martini ${ }^{1,3}$ \\ Accepted: 18 March 2021 / Published online: 1 April 2021 \\ (c) The Author(s), under exclusive licence to Springer Science+Business Media, LLC, part of Springer Nature 2021
}

\begin{abstract}
Coronavirus disease 2019 (COVID-19) has emerged as a pandemic across the world. Hypercoagulability status in COVID-19 is one of the causes of complication from severe COVID-19 with a high risk of arterial thrombosis. Acute Limb Ischemia is a vascular emergency caused by sudden decrease in the arterial perfusion. We report the case of a 53-year-old male patient with COVID-19 Pneumonia, diagnosed with Acute Limb Ischemia. From clinical examination, which included anamnesis, physical examination, and laboratory results as well as chest X-rays, a suspicion of Acute Limb Ischemia was found in a patient with COVID-19 pneumonia. The SARS-CoV-2 real time PCR examination showed positive results. In this patient, the diagnosis of Acute Limb Ischemia with Covid-19 Pneumonia was established through a multidisciplinary approach covering the fields of pulmonology, cardiology, and thoracic and cardiovascular surgery.
\end{abstract}

Keywords Acute limb ischemia $\cdot$ ALI $\cdot$ Hypercoagulability $\cdot$ COVID-19

\section{Highlights}

- The Coronavirus Disease 2019 (COVID-19) is associated with a hyper-coagulability state.

- Both arterial and venous thrombosis can occur in COVID-19 associated hypercoagulability state

- ALI, a vascular emergency can lead to severe morbidity and mortality.

\section{Introduction}

Currently, the COVID-19 pandemic has reached 8,385,440 confirmed cases worldwide with 450,686 deaths in several countries, including Indonesia [1]. Until mid-June 2020, the

Ulfah Kartikasari

ulfahkartika@student.ub.ac.id

Dr Saiful Anwar General Hospital, Malang, Indonesia

2 Department of Pulmonology and Respiratory Medicine, Faculty of Medicine, Brawijaya University, Dr Saiful Anwar General Hospital, Malang, Indonesia

3 Department of Cardiology and Vascular Medicine, Faculty of Medicine, Brawijaya University, Dr Saiful Anwar General Hospital, Malang, Indonesia number of confirmed cases of Covid-19 in Indonesia has reached 43,803 cases with 17,349 cases declared fully recovered and 2373 death cases. The highest number of cases in Indonesia occurred in Jakarta with 9655 cases, followed by East Java with 9057 cases. Data from the Indonesian COVID-19 team reported the characteristics of COVID-19 patients; The case most frequently occurred in the 31-45 age group, followed by 46-59 and 18-30. In COVID-19, the most common comorbid diseases were hypertension (51.6\%), followed by Diabetes Mellitus (35.3\%) and Heart disease (20.6\%) [2].

Acute limb ischemia is one of emergency cases in vascular disease caused by a sudden decrease in arterial limbs perfusion. There are many factors that could cause acute limb ischemia. Thrombotic occlusion is one of atherosclerotic diseases associated with acute limb ischemia. Patients with hypercoagulation state are at risk of arterial thrombosis. Recent research had shown an overall increase in mortality and amputation rate in hospitals. In patients with hypercoagulation state, the decision of surgical intervention must be determined quickly due to the clinical outcome [3].

In this study, we present a case of Acute Limb Ischemia associated with hypercoagulation state in a COVID-19 patient. The aim of this case report is to identify the incidence, characteristics, and clinical outcomes of the treatment of Covid-19 patients with Acute Limb Ischemia. 


\section{Case report}

A 53-year-old male patient with past medical history of uncontrolled hypertension presented to a public health center for dry cough, fever and throat pain of 1 week duration. He was prescribed antipyretic, cough medications and vitamins. A week later, he developed bluish discoloration and worsening pain in bilateral legs with difficulty walking (Fig. 1).

He went to the hospital and underwent a SARS-CoV-2 rapid test which showed a reactive $\operatorname{IgM}$ antibody for SARS-CoV-2. He was admitted and treated by pulmonologist and cardiologist for 4 days and was given antivirals and antibiotics. However, due to worsening Chest X-ray results, he was referred to the central referral COVID-19 hospital.

In the Emergency Room, his condition is hemodynamically stable with BP of $146 / 82 \mathrm{mmHg}$, pulse of $120 \mathrm{bpm}$, Axial temperature of $36.7^{\circ} \mathrm{C}$, and respiratory rate of 20 times/min with $96 \%$ peripheral oxygen saturation on $3 \mathrm{lpm}$ nasal canulla. His extremities were swollen and bluish in color, cold to touch, and had a peripheral oxygen saturation of $74 \%$ (right foot) and $71 \%$ (left foot). Laboratory tests showed leukocytosis, with leukocyte value of 16,380 (normal value: 4700-11,300), hematocrit value of 35.3 (38-42), neutrophilia, valuing 81.2\% (51-67\%), lymphocytopenia, with $8.2 \%$ lymphocytes count (25-33\%) accompanied by increased Neutrophil Lymphocyte Ratio of 9.9 (1-3), monocytosis, valuing $8.7 \%(2-5)$, increased liver function test, with ALT value of $84(0-33)$ and AST value of 37 (0-32), mild hypoalbuminemia of 3.4 (3.5-5.5), Hyponatremia, valuing 130 (136-145), increased CRP value of $4.23(<0.3)$, LDH value of $1101(240-480)$, D-Dimer value of $3.44(<0.5)$, Fibrinogen value of 481.9 (154.3-397.9) and Ferritin value of 786.6 (30-400). Chest $\mathrm{X}$-ray showed decreased infiltrates in both lungs compared with previous CXR. (The improvement of patient's chest $\mathrm{X}$-ray results during hospitalization is shown in Fig. 2).

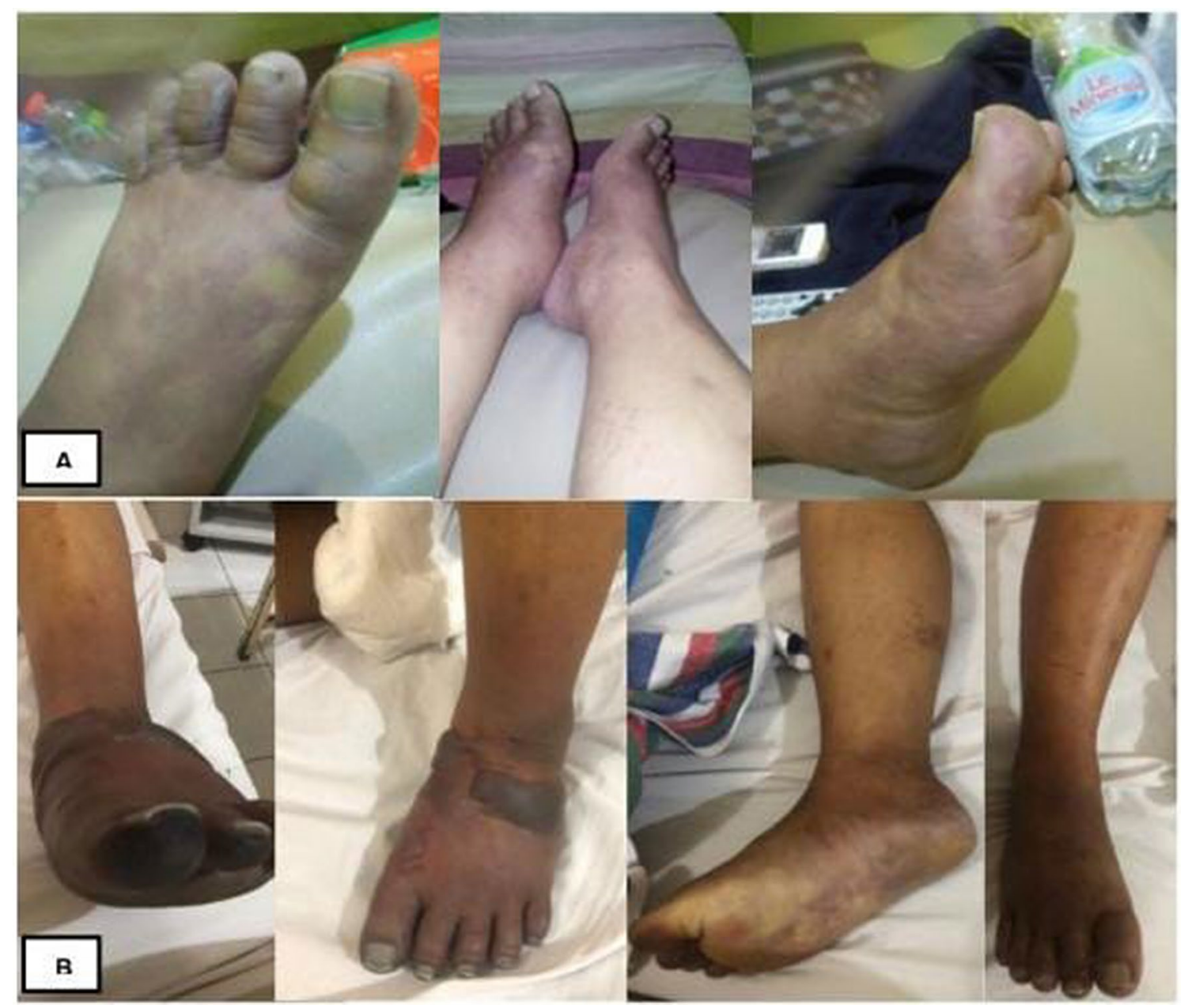

Fig. 1 a Showed ischemia in the patient's limbs on May 16, 2020 before thrombectomy. b Showed both limbs 1 week after thrombectomy 


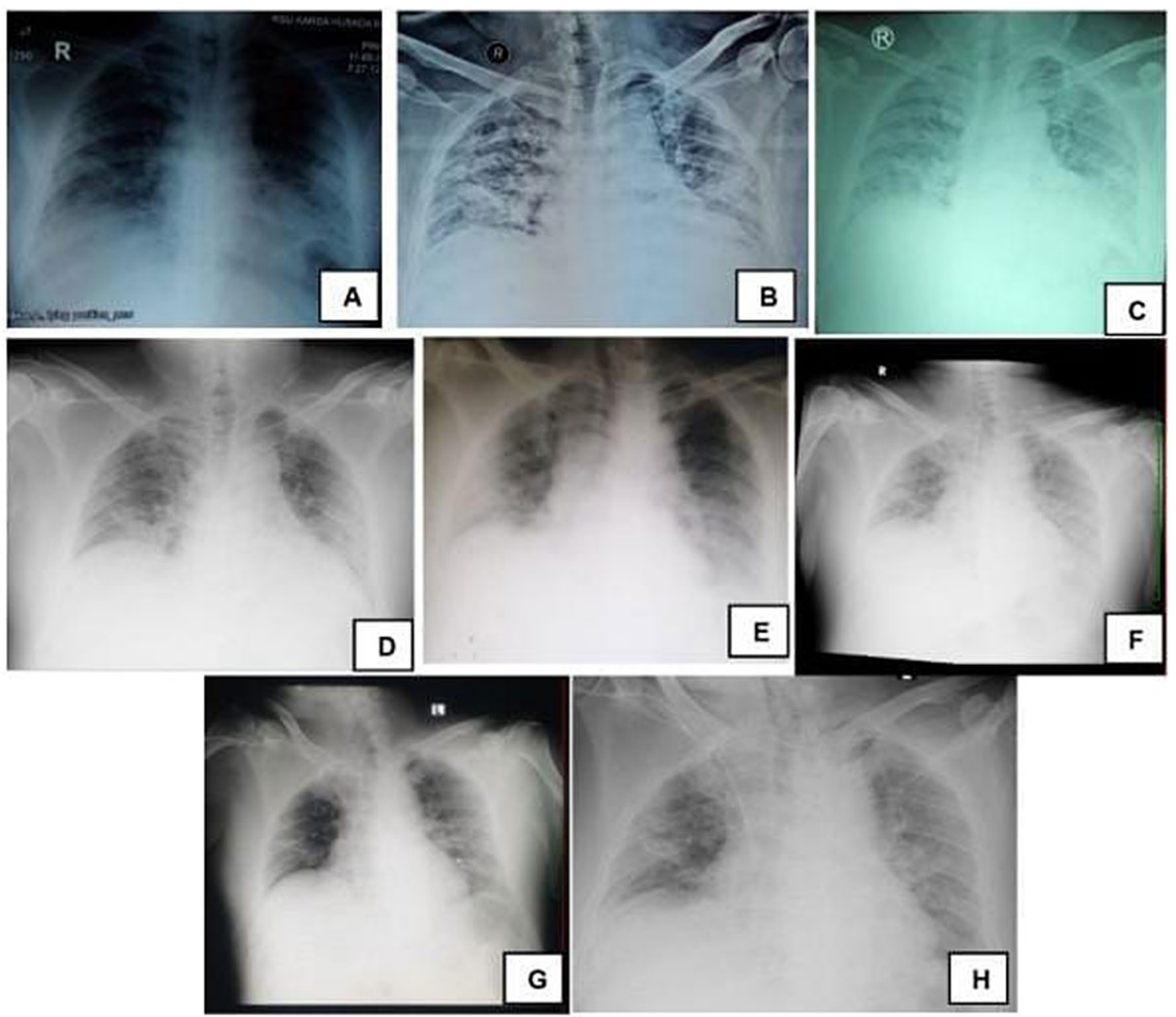

Fig. 2 An overview of the development of chest X-ray during hospitalization from May 11, 2020 to June 2, 2020. a and b Showed the worsening of chest X-ray characterized by the increasing of infiltrate in both pulmonary before referred to the RSSA. $\mathbf{c}-\mathbf{h}$ showed the

He underwent a doppler ultrasound which showed thrombosis of left superficial femoral artery extending to the left popliteal artery and thrombosis of right popliteal artery (Fig. 3). He was consulted to the thoracic and vascular surgery department for thrombectomy. However, the procedure was delayed due to the concern of the patient and his family. He was then admitted in the isolation ward.

The patient underwent a real time PCR examination for SARS-CoV-2 which showed a positive result. Hence, he was diagnosed as confirmed pneumonia Covid-19 case, and were treated with an antibiotic, antiviral, anticoagulant (Enoxaparin), corticosteroids, antiplatelet (clopidogrel), anti-hypertension, anti-cholesterol and vitamins. After 3 days of enoxaparin injection, the cardiologist started heparin therapy (1440 units; dose of 18 units $/ \mathrm{kgBW} / \mathrm{h}$ ) as a replacement for enoxaparin as the antiplatelet medication. improvement of the patient's chest X-ray during hospitalization characterized by decreased infiltrates in gradual terms in the picture of the chest X-ray

He underwent a thrombectomy on May 21st, 2020 and thrombus of length $11 \mathrm{~cm}$ from the left leg and $2 \mathrm{~cm}$ from the right leg were removed (Fig. 4). Patients were treated further in the isolation room with a heparin drip $(15,000$ units/24 h). In conclusion, during his hospitalization the patient was given meropenem for 11 days, moxifloxacin for 10 days, Azithromycin for 7 days, Hydroxychloroquine for 7 days, Oseltamivir for 7 days, Cloxacilin for 7 days, Metronidazole for 7 days and Levofloxacin for 7 days. However, after 1 week of admission, the vascular surgical team proposed an amputation due to lack of improvement after thrombectomy and anticoagulants. The patient refused amputation and was then discharged. 


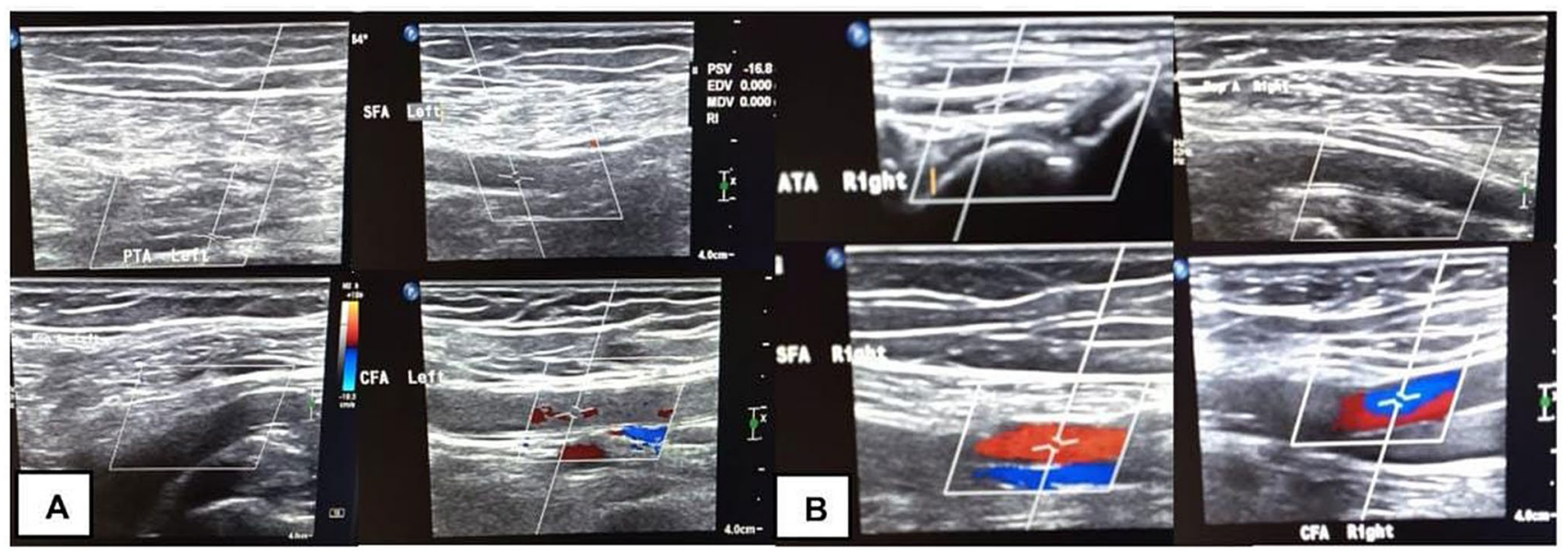

Fig. 3 Doppler Ultrasonography of Left (a) and Right (b) Lower Limbs on May 17, 2020 at dr. Saiful Anwar Hospital Malang indicated the presence of thrombus from left superficial femoral artery (filling $>50 \%$ lumen) to popliteal artery (filling the lumen) with non- detected flow from left anterior tibial artery to posterior tibial artery, as well as thrombus filling right popliteal artery lumen (with undetectable flow); The image indicated blocked flow from anterior to posterior tibial artery
Fig. 4 Thrombectomy process showing left femoral artery (a); thrombectomy process on left femoral artery (b); right femoral artery $(\mathbf{c})$; and a thrombus obtained from the total thrombectomy $(\mathbf{d})$, with a length of $13 \mathrm{~cm}$

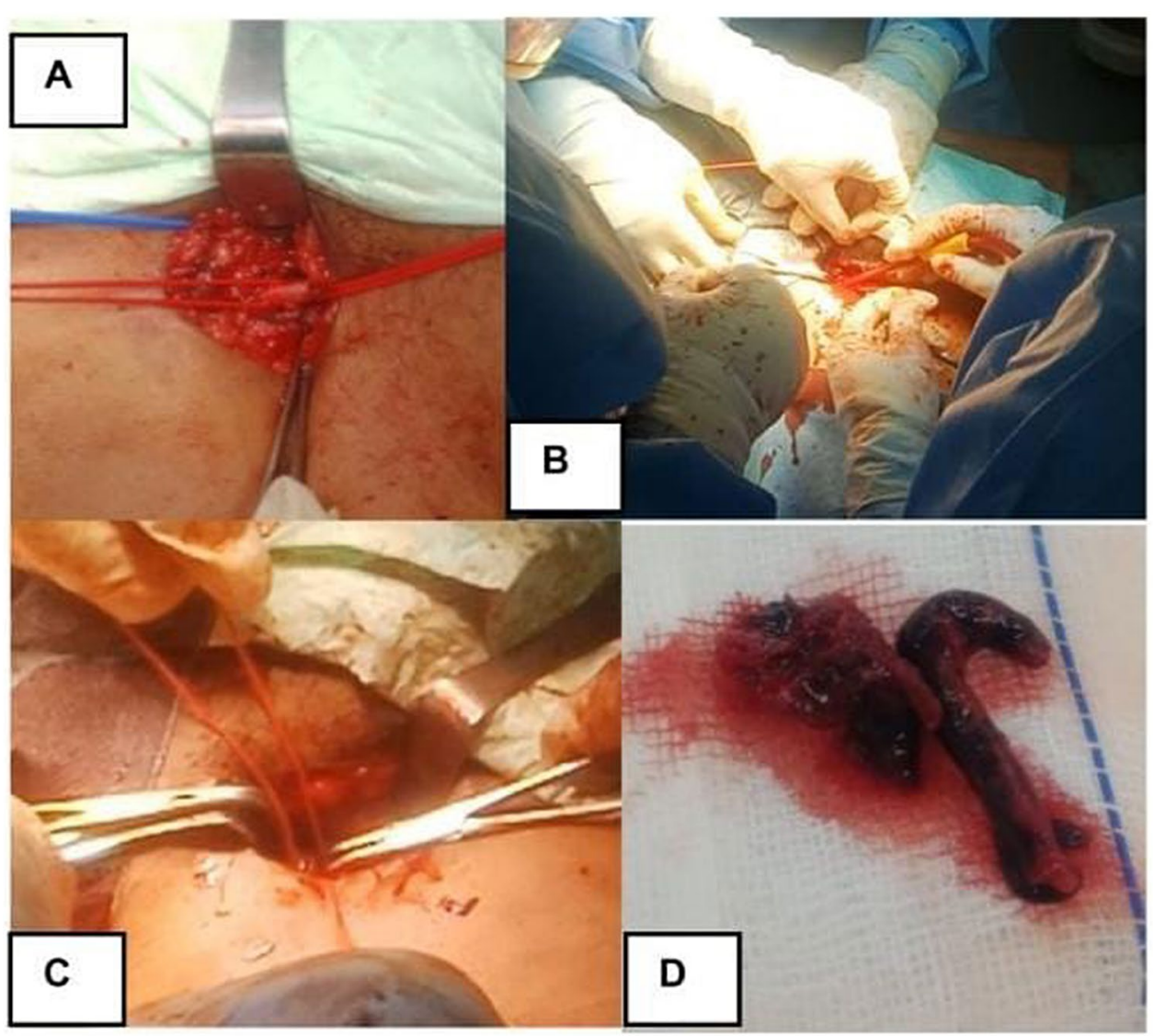

\section{Discussion}

Based on existing literatures, there is a correlation between Covid-19 infections and coagulopathy, which can manifest as acute limb ischemia. This study is expected to increase the understanding of hypercoagulation state in Covid-19 patients and acute limb ischemia as a possible manifestation. Several previous reports with limited clinical and 
therapeutic data have begun to identify Covid-19 patients with incidence of acute limb ischemia [4, 5].

In this case, the patient showed clinical symptoms in the form of pain and bluish rash accompanied by legs that were cold to touch. These are in accordance to a study, in which was mentioned that symptoms of acute limb ischemia are as following: pain, numbness, paresthesia, coldness, and irreversible purpura at extremities [6].

In COVID-19 patients with acute limb ischemia, there is an increase of D-dimer levels, which can also be seen in this case (D-dimer level of 3.44). In addition, it was also stated in a study that 6 out of 7 patients who received anticoagulants, 2 patients were able to survive, and one of them showed improvements in their ischemia. However, in other cases, the ischemia continued to progress into gangrene [5, 7, 8]. A study by Guan et al. [9] showed that patients with high levels of D-dimer tend to have more severe forms of the disease, requiring ICU admission and mechanical ventilation and even lead to death [9].

In addition, Tang et al. also mentioned that patients with Covid-19 pneumonia with significantly higher levels of D-dimer and fibrinogen with prolonged prothrombin time had a higher risk of death compared to patients with normal D-dimers and fibrinogen. In this case, the patient had an increased level of D-dimer $(3.44 \mathrm{mg} / \mathrm{mL})$ and fibrinogen (481.9). Based on the clinical conditions, it can be presumed that the hypercoagulation state with clinical ischemia in this patient signified severe illness and required close monitoring and appropriate early intervention management. Close monitoring of the extremities for thromboembolism is needed to avoid permanent damage or possible amputation $[10,11]$. Elevated D-dimer levels had also been shown to be directly related to the severity of the hypercoagulable state in Covid19 patients [12].

Other than D-dimers, platelet count was also examined in this patient to assess the presence of hemoconcentration at the early weeks of treatment. In addition, the patient's laboratory examination also showed neutrophilia, lymphocytopenia, hypoalbuminemia, increased liver enzymes, LDH, and procalcitonin, as well as CRP. This is in accordance with a study by Lippi and Plebani, which summarized several studies in Wuhan regarding laboratory abnormalities in patients with Covid-19 infection; Among them there were a decrease in lymphocyte count and albumin as well as an increase of WBC, neutrophils, LDH, liver enzymes, bilirubin, creatin, troponin enzymes, D-dimer, Prothrombin time, procalcitonin and CRP [13, 14].

The patient's CXR showed infiltrate, consistent of pneumonia. Ackermann et al., in their study, stated that in COVID-19 pneumonia, histological examination would typically show a depiction of severe alveolar damage associated with T-cell infiltration in the perivascular spaces, and in addition, extensive thrombosis accompanied by microangiopathy. The study also mentioned that angiogenesis of Covid-19 pneumonia patients' lungs was 2.7 times as high as that of influenza patients [15].

In this case, the patient underwent thrombectomy, followed by anticoagulation therapy. After thrombectomy and anticoagulation therapy, the patient showed clinical improvement within 3 days. However, within the first week, gangrene-an indication for an amputation-occurred. This phenomenon could occur either due to medical and nonmedical factors; Non-medical factors including the difficulty to monitor the administration of intravenous anticoagulants associated with commonly difficult intravenous entry access, and difficulty in monitoring hemostatic parameter (measured every $8 \mathrm{~h}$ ) making it difficult to achieve target hemostatic parameters with the aforementioned anticoagulants. Thrombectomy was indicated in accordance to this patient stage being Rutherford stage II. Survival without amputation at 12 months was better after thrombectomy than cathetertargeted thrombolysis or surgical thrombectomy [16].

On the first 3 days of evaluation after thrombectomy and anticoagulant therapy, the patient showed increased tissue vascularization, as indicated by the presence of popliteal and femoral artery pulses in both limbs. Therefore, proper administration of anticoagulants is highly recommended for the treatment of acute limb ischemia, and prophylactic anticoagulant administration should be considered. Another suspected cause of ischemic cases is the use of vasopressor; However, vasopressor administration does not cause unilateral ischemia. Another possible cause of acute limb ischemia is the presence of emboli. The presence of thrombus in femoral and popliteal arteries in this patient can lead to the clinical symptoms of ischemia $[6,16,17]$. Lovenoxa prophylactic anticoagulant indicated in COVID-19 patients with coagulopathy-administered in this patient gave no significant improvement, therefore the need to proceed with thrombectomy [10].

The patient was given Oseltamivir, an inhibitor of viral neuraminidase. This decision to administer oseltamivir for compassionate use was based on the current drug availability. However, Sanders et al. reported that oseltamivir had no positive effect in the management of COVID-19 without influenza A or B viruses co-infection [17]. Other antiviral drugs could not be prescribed within the research time window, and oseltamivir was the only drug available. Antibiotic therapy given to the patient was in accordance to empirical antibiotic therapy at the beginning of treatments and followed by specific antibiotic therapy based on drug sensitivity test. Antibiotic replacement was performed due to the worsening condition of this patient during treatment.

Zhang et al. had recently suggested an antiphospholipid antibody examination as a contributor or possible cause of thrombotic occurrence. Although the patient's antiphospholipid antibody was not examined in this study, this hypothesis 
may provide a potential explanation for the occurrence of ischemia. The relationship between coagulopathy and poor prognosis in Covid-19 patients need to be discussed. Several studies have shown that patients with abnormal coagulation parameters with clinical signs of limb ischemia were at higher risk for a worse prognosis $[1,6,7,12]$.

Funding Funding support was provided by the Faculty of Medicine, Brawijaya University and r Saiful Anwar General Hospital.

\section{Declarations}

Conflict of interest The authors declares that they have no conflict of interest.

Ethical approval The patient gave permission and informed consent for the publication of this case report.

\section{References}

1. John Hopkins University and Medicine (2020) Coronavirus Resources Centre. https://coronavirus.jhu.edu/map.html. Accessed 14 Aug 2020

2. Gugus Tugas Percepatan Penanganan Covid-19 (2020) Data Covid-19. https://covid19.go.id/. Accessed 14 Aug 2020

3. Lindholt JS (2018) 2017 ESC guidelines on the diagnosis and treatment of peripheral arterial diseases in collaboration with the European Society for Vascular Surgery (ESVS). Eur Heart J 39:763-821

4. Zhang Y, Cao W, Xiao M et al (2020) Clinical analysis of seven cases of critical new coronavirus pneumonia complicated with extremity ischemia. Zhonghua xue ye xue za zhi = Zhonghua Xueyexue Zazhi. https://doi.org/10.3760/cma.j.issn.0253-2727. 2020.008

5. Zhang Y, Xiao M, Zhang S et al (2020) Coagulopathy and antiphospholipid antibodies in patients with Covid-19. N Engl J Med 382(17):e38
6. Obara H, Matsubara K, Kitagawa Y et al (2020) Acute limb ischemia. Ann Vasc Dis 11(4):443-448

7. Levi M, Thachil J, Iba T et al (2020) Coagulation abnormalities and thrombosis in patients with COVID-19. Lancet Haematol. https://doi.org/10.1016/S2352-3026(20)30145

8. Lai CC, Ko WC, Lee PI et al (2020) Extra-respiratory manifestations of COVID-19. Int J Antimicrob Agents 56(2):106024. https://doi.org/10.1016/j.ijantimicag.2020.106024

9. Guan W, Ni Z, Hu Y et al (2020) Clinical characteristics of 2019 novel coronavirus infection in China. Respir Med 382:1708-1720. https://doi.org/10.1056/NEJMoa2002032

10. Tang N, Li D, Wang X, Sun Z et al (2020) Abnormal coagulation parameters are associated with poor prognosis in patients with novel coronavirus pneumonia. J Thromb Haemost 18(4):844-847

11. Han H, Yang L, Liu R et al (2020) Prominent changes in blood coagulation of patients with SARS-CoV-2 infection. Clin Chem Lab Med 58(7):1116-1120

12. Lippi G, Favaloro EJ (2020) D-dimer is associated with severity of coronavirus disease 2019: a pooled analysis. Thromb Haemostas. https://doi.org/10.1055/s-0040-1709650

13. Lippi G, Plebani M (2020) Laboratory abnormalities in patients with COVID-2019 infection. Clin Chem Lab Med 58(7):1131-1134

14. Becker RC (2020) COVID-19 update: Covid-19-associated coagulopathy. J Thromb Thrombol 2020(50):54-67

15. Ackermann M, Verleden SE, Kuehnel M et al (2020) Pulmonary vascular endothelialitis, thrombosis, and angiogenesis in Covid19. N Engl J Med 2020(383):120-128. https://doi.org/10.1056/ NEJMoa2015432

16. Landry GJ, Mostul CJ, Ahn DS et al (2018) Causes and outcomes of finger ischemia in hospitalized patients in the intensive care unit. J Vasc Surg 68(5):1499-1504

17. Olinic DM, Stanek A, Tataru DA et al (2019) Acute limb ischemia: an update on diagnosis and management. J Clin Med 8:1215. https://doi.org/10.3390/jcm8081215

Publisher's Note Springer Nature remains neutral with regard to jurisdictional claims in published maps and institutional affiliations. 